Résumés des conférences et travaux

\title{
Initiation à l'histoire de la gravure
}

José Lothe

\section{(2) OpenEdition}

Journals

Édition électronique

URL : https://journals.openedition.org/ashp/742

DOI : 10.4000/ashp.742

ISSN : 1969-6310

Éditeur

Publications de l'École Pratique des Hautes Études

\section{Édition imprimée}

Date de publication : 1 octobre 2009

Pagination : 287-303

ISSN : 0766-0677

\section{Référence électronique}

José Lothe, «Initiation à l'histoire de la gravure », Annuaire de l'École pratique des hautes études (EPHE) Section des sciences historiques et philologiques [En ligne], 140 | 2009, mis en ligne le 20 octobre 2009, consulté le 06 juillet 2021. URL : http://journals.openedition.org/ashp/742 ; DOI : https://doi.org/ 10.4000/ashp.742 


\title{
INITIATION À L'HISTOIRE DE LA GRAVURE
}

\author{
Maître de conférences : M. José Lothe
}

Programme de l'année 2007-2008 : I. L'œuvre gravé de Marcantonio Raimondi. - II. Les estampes d'après Titien. - III. Édition des Notes manuscrites de P.-J. Mariette, correspondant à la collection d'estampes du prince Eugène de Savoie (suite).

Au cours des années précédentes, le maître de conférences avait fait des estampes gravées par Marcantonio Raimondi d'après Raphaël à partir de 1511 jusqu'au sac de Rome en 1527, l'objet de son enseignement; cependant, excepté des notices dans des catalogues d'exposition ${ }^{1}$, l'œuvre de Raimondi dont Vasari a longuement parlé n'a été répertorié que par Henri Delaborde dans un catalogue publié en $1888^{2}$ qui sert toujours de référence. Il a donc paru nécessaire de constituer un nouveau catalogue de toutes les estampes de Raimondi et ce fut le premier thème des conférences. D'autre part, les conférences de l'année 2006-2007 ayant eu pour sujet les premières estampes produites à Venise, celles de l'année 2007-2008 se sont tout naturellement intéressées aux planches gravées d'après Titien. L'ampleur des deux sujets a fait que le temps a manqué et, le maître de conférences ayant dû prendre sa retraite, ils ne pourront être menés à leur terme.

L'essentiel de la carrière de Marcantonio s'est déroulé à Rome. Toutefois ce ne fut pas dans la Ville éternelle qu'il découvrit et apprit l'art de la taille-douce, où vraisemblablement elle était peu développée lorsqu'il y arriva en 1511. Il était né à San Andrea in Argene, ville située à une vingtaine de kilomètres de Bologne. Faute de documents ${ }^{3}$, on ne peut qu'avancer des hypothèses sur la date de sa naissance, échelonnée selon les auteurs entre 1470 et 1488 . Le témoignage de l'humaniste Giovanni Filoteo Achilini, dont Raimondi grava le portrait ${ }^{4}$, permet cependant, nous semble-t-il, de placer sa naissance au plus tard en 1480. En effet, dans son poème $I l$ Viridario, composé en 1504 et publié en 1513, Achilini a consacré six vers à Marcantonio :

«Consacro anchor Marc Antonio Raimondo

« Che imita de gli antique le sante arme

1. En particulier les catalogues des expositions The engravings of Marcantonio Raimondi, The University of Kansas, 1982; Bologna e l'Umanesimo, 1490-1510, Bologne, 1998; Roma e lo stile classico di Raffaello. 1515-1527, Milan, 1999.

2. Delaborde (Henri), Marcantonio Raimondi. Étude historique et critique, suivie d'un catalogue raisonné des cuvres du maître, Paris, 1888.

3. Rappelons qu'une édition d'Aulu-Gelle sortie des presses aldines en 1515 porte la mention qu'elle avait appartenu à Raimondi et, auparavant, à son père André. D'autre part une lettre datée du 10 mars 1659 et expédiée de Bologne à François Langlois indiquait qu'à cette date un libraire prétendait descendre d'un bâtard de Marc-Antoine et qu'il possédait quatre cuivres et un livre de dessins du graveur. Cf. Benjamin Fillon, « Nouveaux renseignements sur Marc-Antoine Raimondi », dans Gazette des Beaux-Arts, mars 1880, p. 229-238.

4. The Illustrated Bartsch (écrit TIB par la suite), vol. 27, p. 141. 
«Col designo, e bollin molto et profondo

«Come se veden sue vagnhe e riche forme

«Hamme rettratto in rame come io scrivo

«Ch'en dubio de noi pende, quale e vivo ${ }^{1}$.

C'était un apprentissage long et méticuleux que celui du burin et il est difficilement concevable qu'un artiste, âgé de seize ans s'il était né en 1488, ait pu mériter un tel éloge. D'autre part, si on en croit Vasari selon qui Raphaël avait donné à l'un des quatre porteurs de la seda gestatoria dans la fresque d'Héliodore chassé du temple datée de 1512, les traits de Marcantonio, il faut convenir qu'il y apparaissait comme un homme ayant bien dépassé la trentaine. Il est donc loisible de penser que Marcantonio n'était pas né après 1480 . Sa formation se fit d'abord à Bologne et, toujours selon Vasari, son premier maître fut Francesco Raibolini, surnommé Francia ${ }^{2}$, peintre, orfèvre et nielleur dont le poète Angelo Michele Salimbeni avait loué en 1487 les talents dans son Epitalamii pro nuptiali pompa Anibalis Bentivioli, n'hésitant pas à écrire qu'il était supérieur à Maso Finiguerra ${ }^{3}$, le premier graveur en taille-douce florentin. S'il s'est plié aux usages de l'époque, Raimondi était âgé d'une quinzaine d'années environ lorsqu' il commença de travailler avec Francia et son arrivée à Bologne dut se faire au plus tard vers 1495 .

On a vu Achillini louer les talents de Raimondi et se réjouir du portrait que le graveur avait fait de lui. Poète, écrivain, musicien et curieux de l'Antiquité, Achillini appartenait au cercle intellectuel et humaniste qui s'était constitué à Bologne et qui réunissait des hommes comme Giovanni Battista Pio et Filippo Beroaldo, philologues l'un et l'autre et éditeurs de textes antiques, Angelo Michele Salimbini. Faisaient également partie de ce cercle Francesco Francia et le dessinateur et peintre Amico Aspertini. Raimondi séjourna à Bologne jusqu'en 1506; très proche de ce groupe il fut particulièrement lié avec Francia d'après qui il grava de nombreuses estampes, allégoriques et souvent difficiles à interpréter faute de textes qui en donneraient les clefs, ou inspirées par la mythologie ou encore l'histoire antique. Lorsqu'il a signé ses planches, Raimondi se servit du monogramme MAF (Marc Antonio Fecit) dont la graphie prit différentes formes, la plus fréquente toutefois étant celle où les trois lettres étaient accolées.

Les estampes ayant la faculté de circuler facilement, Raimondi eut l'occasion de voir les œuvres d'Albert Dürer qu'il rencontra peut-être à Bologne en octobre $1506^{4}$, au cours du second voyage de l'artiste en Italie. Le regard que l'Italien porta sur les œuvres de l'Allemand et l'usage qu'il en fit montrent la complexité des rapports entre les deux

1. Voici la traduction : Je rends aussi hommage à Marcantonio Raimondi/ Qui a mis ses pas dans ceux des maîtres antiques/ Et qui est autant habile dans le dessin et dans le burin/ Qu'il est précis dans ses belles planches gravées/ Comme je l'écris il a fait un portrait de moi sur cuivre/ Et je ne sais lequel est le plus vivant.

2. Né à Bologne en 1450, mort le 5 janvier 1517.

3. Ma fra gi orafi io diro il Franza/ Che io non lo scio lasciar per maggior cura,/Lui Polygnoti con il pennello avanza/ E Phidia all'operar della sculptura, / E col bullino ha tanta nominanza/ Che la sua a Maso Finiguerra oscura/ A Costui fo comparation di morti/Perche che viva invidia al ver non porti. Cité par D. Landau et P. Parshall, The Renaissance Print. 1470-1550, p. 99.

4. Dans une lettre adressée à son ami Pirckheimer et datée aux alentours du 13 octobre 1506, Dürer faisait part de son intention de se rendre à Bologne pour étudier la perspective. 
hommes. D'une part, Marcantonio fut sans doute celui qui fit le plus de planches copiées sur des estampes de Dürer : nous en avons compté soixante-huit, nombre élevé en raison des planches de la Vie de la Vierge, copiée presque entièrement, et de celles de la Petite Passion. D'autre part, Raimondi plaça souvent dans ses propres estampes, en arrière plan, des paysages pris dans des œuvres de Dürer. Il y a davantage : jusqu'à ce que Dürer s'en émût et portât l'affaire devant le Sénat de Venise, Raimondi n'hésita pas à signer ses propres copies du monogramme $A D$, montrant par là son désir que la renommée du graveur allemand leur profitât.

Les premières œuvres de Dürer que Raimondi copia à Bologne constituaient un ensemble de six burins gravés entre 1495-1496 et 1498-1499. C'était la Sainte Famille au papillon ${ }^{1}$, Les Offres d'amour ${ }^{2}$, appellation pudique d'une estampe représentant un homme âgé prêt à vider sa bourse pour monneyer les faveurs d'une jeune dame, Les Amants guettés par la Mort ${ }^{3}$, les Trois paysans ${ }^{4}$, La Dame et le lansquenet ${ }^{5}$ et le Songe du paresseux ${ }^{6}$. Nous datons ces copies des années 1500. En les gravant, Raimondi s'est attaché à reporter le plus exactement possible sur ses propres planches les compositions de Dürer dont il a suivi scrupuleusement les tailles et dont il a repris les dimensions. C'eût été des copies parfaites sauf que Raimondi ayant dessiné et gravé les œuvres telles qu'il les voyait, les estampes, une fois imprimées, se trouvaient inversées. Pourtant il les signa du monogramme $A D$ et si on ne peut douter que Raimondi a voulu connaître parfaitement, tant pour son dessin que pour sa propre pratique du burin, les manières de dessiner et de graver de Dürer, on doit convenir que par la même occasion ses estampes se substituaient aux œuvres originales.

Nous ne saurions dire si ce fut à Bologne ou à Venise où il s'était rendu à la fin de l'année 1506 que Marcantonio découvrit les gravures en bois de Dürer. Elles présentaient des traits plus faciles à suivre, plus commodes à transposer sur les cuivres que ceux des burins, et si l'on place côte à côte les estampes originales et leurs copies, il est manifeste que Raimondi a transcrit avec son propre burin, taille après taille, le travail de gravure de Dürer. Il copia d'abord, pensons-nous, la planche représentant la Déploration, tirée de la suite de la Grande Passion ${ }^{7}$. Exécutée par Dürer circa 14981499, elle fut superbement traduite en taille-douce par Raimondi dont la copie peut être regardée comme une véritable re-création de l'originale; elle avait été toutefois dessinée dans le même sens sur le cuivre et l'impression se trouvait inversée. Les autres bois devenus tailles-douces, La Vierge allaitante ${ }^{8}$, Saint Jean Baptiste et saint Onuphre dans le désert ${ }^{9}$, Saint François recevant les stigmates ${ }^{10}$ et Saint Christophe ${ }^{11}$

1. TIB, vol. 10 , p. 41 et $T I B$, vol. 27, p. 326 .

2. TIB, vol. 10, p. 80 et $T I B$, vol. 27, p. 335 .

3. $T I B$, vol. 10 , p. 81 et $T I B$, vol. 27 , p. 336 .

4. TIB, vol. 10, p. 75 et $T I B$, vol. 27, p. 334 .

5. $T I B$, vol. p. 73 et $T I B$, vol. 27, p. 334 .

6. $T I B$, vol. 10 , p. 000 et $T I B$, vol. 27 , p. 336.

7. H. Delaborde assure que Raimondi grava aussi La Cène ( ${ }^{\circ} 296$ de son catalogue) mais nous n'en connaissons aucune d'épreuve.

8. TIB, vol. 10 , p. 194 , et $T I B$, vol. 27 , p. 325

9. TIB, vol. 10 , p. 207 et TIB, vol. 27 , p. 329.

10. $T I B$, vol. 10 , p. 205 et $T I B$, vol. 27 , p. 328.

11. TIB, vol. 10 , p. 199 et $T I B$, vol. 27 , p. 327. 
furent différents dans la mesure où le dessin de Raimoni inversait les compositions de sorte qu'une fois imprimées les estampes étaient dans le même sens. Gravés vers 1503-1504, les originaux faisaient partie d'une série qui n'avait été qu'exécutée dans l'atelier de Dürer mais Raimondi l'ignorait et, tout autant que pour les burins, il comptait bien, en proposant au public des copies cette fois tout à fait conformes et signées de son monogramme, en tirer bénéfice.

Nous avons dit plus haut qu'en se rendant à la fin de l'année 1506 à Venise où il séjourna jusqu'en 1508, Raimondi donna à sa carrière une nouvelle orientation. En ces premières années du nouveau siècle, la taille-douce était essentiellement représentée à Venise par Jacopo de Barbari qui, toutefois, avait quitté sa patrie pour aller travailler dans les cours allemandes, et par Giulio Campagnola. A-t-il été attiré dans la cité maritime par la réputation de Bellini ou par celle de Giorgione encore qu'en ces temps peintures et estampes ne fussent pas liées? Certes on le voit graver une estampe inspirée par Giorgione et par Giulio Campagnola, dite le Songe de Raphaël ${ }^{1}$ appellation impropre pour une estampe dont la signification échappe toujours. Une autre de ses estampes, le Saint Jérôme au petit lion ${ }^{2}$, avait pour source un dessin de Titien mais il est possible qu'il ait copié l'œuvre d'Agostino Veneziano. Avait-il l'intention de profiter du développement de la gravure en bois, florissante à Venise? De fait, on le voit graver en bois une Incrédulité de saint Thomas ${ }^{3}$ utilisée plus tard pour illustrer un ouvrage $^{4}$. On ne peut que constater qu'il grava très peu de tailles-douces au cours de son séjour vénitien et qu'il continua de copier des bois de Dürer. Dans les lignes qu'il a écrites sur son séjour vénitien, Vasari s'est trompé en affirmant que Marcantonio avait acheté des épreuves de la Petite Passion afin d'en graver des copies. Le graveur eut été bien en peine de le faire puisque Dürer n'a gravé cette suite qu'entre 1509 et 1511. En réalité les planches copiées à Venise furent celles de la Vie de la Vierge, du moins les dix-sept gravées par Dürer entre 1502 et $1505^{5}$. C'était certainement une commande des libraires vénitiens Niccolo et Domenigo dal Gesu dont on peut voir la marque sur la planche du Couronnement de la Vierge. À l'instar des copies qu'il avait faites auparavant à Bologne, Raimondi n'hésita pas à les signer du monogramme de Dürer. Toutefois Raimondi avait de nouveau pris soin d'inverser sur ses cuivres les compositions de Dürer et les estampes imprimées retrouvaient le sens des originaux. Ainsi l'illusion était parfaite et les copies pouvaient être regardées et vendues comme des estampes de Dürer. Face à ces pratiques, on comprend l'émotion de Dürer qui, toujours selon Vasari, en fut informé alors qu'il se trouvait au Pays-Bas d'où il retourna à Venise pour porter l'affaire devant le Sénat ${ }^{6}$. Le jugement rendu obligeait Raimondi à ne pas signer du nom de Dürer les copies des estampes qu'il pourrait encore faire. Il s'exé-

1. TIB, vol. 27, p. 55 .

2. TIB, vol. 26 , p. 133.

3. La planche est signée du monogramme MAF. BNF Est., Eb $5 \mathrm{~d} 4^{\circ}$. Delaborde, Marc-Antoine Raimondi, $\mathrm{n}^{\circ} 310$.

4. Gravée pour l'ouvrage intitulé Epistole \& euangeli volgari hystoriade, imprimée à Venise en 1512 pour Nicolo et Domenigo dal Gesu.

5. Les deux planches de la Mort de la Vierge et de L'Assomption furent gravées en 1510; celle du frontispice en 1511.

6. Vasari, Le Vite..., éd. Chastel, t. 7, p. 66. 
cuta lorsque, installé à Rome, il grava des copies des trente-sept planches de la Petite Passion, qui imprimées procuraient des images dans le même sens qu'il signa d'une tablette blanche. Il apparaît cependant que Vasari s'est trompé et même s'est contredit; il a confondu les copies de la Vie de la Vierge, gravées les premières et signées du monogramme de Dürer, avec celles de la Petite Passion. À propos de ces planches, il a d'abord écrit que Dürer s'entendit avec Marcantonio pour les éditer et, plus loin, que Dürer fut fort mécontent qu'elles aient été copiées. En outre, si Dürer avait séjourné dans les Pays-Bas en juillet-août 1520, il n'en revint pas pour autant à Venise à cette date. Ceci conduit à s'interroger sur le jugement rendu par le Sénat de Venise et nous devons reconnaître que ce document, abondamment cité, n'est en réalité connu que par l'affirmation de Vasari. On ne peut guère mettre en doute que le Sénat ait rendu un jugement plutôt favorable à Dürer mais il était loin de reconnaître et de protéger la propriété intellectuelle et artistique. On doit constater encore que si Raimondi copia alors qu'il était à Rome deux autre bois de Dürer datés de l'année 1511, sur sa planche de la La Messe de Saint Grégoire ${ }^{1}$ (fut-ce un oubli involontaire de l'arrêt du Sénat ou le désir de passer outre ?) il mit de nouveau le monogramme de Dürer tandis qu'il laissait la planche de L'Adoration des Mages ${ }^{2}$ tout à fait anonyme.

On peut se demander pour quelles raisons Raimondi n'est pas resté à Venise où le milieu artistique était brillant. Sans doute la renommée de Raphaël l'a attiré à Rome et peut-être Francia l'avait-il chaudement recommandé au peintre avec qui il correspondait $^{3}$. Toutefois, avant de se rendre dans la Ville éternelle, Marcantonio était passé par Florence où, grâce à Maso Finiguerra, à Baccio Baldini et à Antonio Pollaiuolo, la tailledouce avait connu un essor extraordinaire. Il put voir aussi le carton de Michel-Ange préparatoire à la fresque de La Bataille de Cascina peinte dans la salle du conseil du palais Vecchio ${ }^{4}$ de Florence, maintenant perdue ${ }^{5}$. À partir des personnages de la Bataille il grava deux estampes qui montraient deux hommes nus dans des postures différentes ${ }^{6}$, l'un assis et enfilant ses chausses, l'autre debout et prenant pied sur un talus. Plus tard, en 1519, Raimondi grava une autre estampe, tirée de la même fresque, dans laquelle il ajouta deux autres hommes, l'un assis, l'autre penché. Dès son arrivée à Rome en 1511, on le voit graver deux estampes dont les modèles se trouvaient dans deux fresques du plafond de la chapelle Sixtine, Adam et Ève chassés du paradis ${ }^{7}$ et L'Ivresse de Noé $^{8}$. Comme dans les précédentes, il porta toute son attention au mouvement des corps et à la musculature des personnages, ne prenant que deux des personnages de l'Ivresse et réduisant la scène de l'expulsion du paradis aux seuls parents du genre humain?

1. TIB, vol. 10 , p. 218 et $T I B$, vol. 27 , p. 330 .

2. TIB, vol. 10 , p. 98 et $T I B$, vol. 27, p. 324.

3. Ainsi en septembre 1508 Raphaël avait écrit à Francia.

4. Dans la même salle les autorités florentines avaient demandé à Léonard de Vinci de peindre la bataille d'Angiari.

5. Le carton est connu par une copie anonyme conservée au British Museum et reproduite dans le catalogue de l'exposition Leonardo da Vinci, Michelangelo and the Renaissance in Florence, Ottawa 2005.

6. $T I B$, vol. 27 , p. 144 et 162 .

7. $T I B$, vol. 26 , p. 10 .

8. TIB, vol. 27 , p. 134

9. Raimondi n'a pris que les deux figures de Sem et Japhet à qui, dans la fresque, leur frère Cham montrait la nudité de leur père. Amputée de cette façon, la scène n'a pas été reconnue, même par les auteurs du 
Après Bologne, après Venise, après Florence, Marcantonio vint à Rome où il était installé au cours de l'année 1510 et où Raphaël était arrivé deux années auparavant. Une des premières planches qu'il grava fut celle d'une estampe où quelques auteurs ont cru pouvoir reconnaître un Triomphe de Scipion ${ }^{1}$ dont le dessin original était l'œuvre de Jacopo Ripanda qui avait été son condisciple à Bologne dans l'atelier de Francesco Francia. Ami du sculpteur Andrea Bregno qui lui laissa sa collection d'antiquités, proche du cardinal Raffaele Riario, lui-même ami de Pomponio Leto, fondateur de l'Accademia romana, Ripanda fut en mesure de faire connaître à toutes ces personnalités romaines les talents de Marcantonio et Raphaël ne pouvait manquer d'en être bientôt informé. Un autre homme, Baviero di Carroci, originaire de Parme, surnommé il Baviera, lui a fourni aussi l'occasion de rencontrer le graveur. Connu essentiellement par les quelques lignes que Vasari lui a consacrées, commis de Raphaël, Baviera montrait grand intérêt pour les estampes, soutint plus tard la vocation de Marco Dente et fit faire à Giovanni Jacopo Caraglio des planches d'après le Rosso. Selon l'auteur des Vite, Raphaël confia dès 1510 à Baviera ${ }^{2}$ de faire graver par Raimondi quelques-uns de ses dessins ${ }^{3}$. Les relations entre Raphaël, Baviera et Raimondi sont confirmées par la présence et la signature de Raimondi le 8 novembre1515, en tant que témoin, sur l'acte d'achat d'une maison ${ }^{4}$ par le peintre représenté par son commis. Si l'on peut considérer comme certain que Raphaël confia à son commis d'organiser matériellement et au mieux de ses intérêts la gravure des dessins, l'impression des planches et la vente des estampes et ceci est corroboré par le fait qu'à la mort du peintre les cuivres devinrent sa propriété, il faut cependant reconnaître que le nom de Baviera n'a été inscrit sur aucune des estampes gravées par Raimondi. D'autre part aucun document n'indique que le peintre ait surveillé l'interprétation gravée de ses dessins ou de ceux provenant de son atelier, comme l'avait fait Andrea Mantegna avec Gian Marco Cavalli ${ }^{5}$. Il faut remarquer aussi que le nom de Raphaël et celui de Raimondi n'ont été inscrits que sur

TIB. Pourtant le sujet avait été identifié par le rédacteur des Notes manuscrites, t. 8, fol. 75 : « Deux hommes presque nuds s'entrenans ensemble; l'on prétend que cette pièce qui est d'un fort bon goût de dessein est de l'invention et de la graveure de Marc-Antoine. Si elle est de luy comme il y a toute apparance, elle approche de ses premières manières... Je crois d'après Michel-Ange et que ce groupe est tiré d'un des tableaux de la chapelle Sixte où est représentée l'ivresse de Noé ».

1. TIB, vol. 26, p. 212.

2. Vasari, éd. Chastel, t. 7, p. 70 : «Raphaël avait chez lui depuis plusieurs années, pour broyer ses couleurs, un aide d'atelier nommé Baviera qui avait quelque expérience. Il décida que Marc-Antoine graverait les planches et que Baviera les imprimerait ».

3. La première estampe gravée d'après Raphaël fut vraisemblablement une Lucrèce (TIB, vol. 26, p. 188) et le même dessin fit naître ensuite une Didon (TIB, vol. 26, p. 181).

4. J. Shearman, Raphael in early modern sources, ouvr. cit., p. 214-215.

5. Cf. A. Canova, Gian Marco Cavalli incisore per Andrea Mantegna e altre notizie sull'ireficeria e la tipografia a mantova nel XV seculo, dans Italia Medioevale e unmanistica, 2001, XLII, p. 149-179. On pourrait citer aussi le contrat passé le 24 octobre 1481 entre Bramante, l'orfèvre Prevaderi et le peintre Mateo de Fideli. Il a été découvert par Luca Beltramonti et a fait l'objet d'un article « Bramante e Leonardo praticarono l'arte del bulino? Un incisore sconosciuto : Bernardo Prevedari », publié dans Rassegna d'Arte, 17 (1917), p. 187-194. Rédigé en latin, cet acte a été de nouveau publié (en partie seulement) dans l'ouvrage, The Renaissance Print, p. 386, note 9. 
sept estampes ${ }^{1}$. D'autres planches n'ont été signées que de la fameuse tablette, considérée comme la marque personnelle de Raimondi, copiée sur celle de Dürer. D'autres - presque toutes des copies - sont signées du monogramme $M A F$ et d'autres enfin ont été gravées anonymement, l'absence de toute signature étant incompréhensible sur des

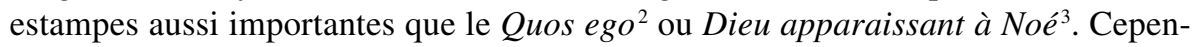
dant, toujours selon Vasari, les estampes furent une source de revenus considérables pour Raphaël. Vraisemblablement, Raimondi devint ainsi le premier graveur en tailledouce à qui furent confiés des dessins du peintre afin d'en produire des copies multiples. L'intérêt de Raphaël pour les estampes a été reconnu par une source tardive, une lettre de Dominicus Lampsonius écrite à Vasari le 25 avril 1565 où il disait son regret que désormais personne n'encourageait plus les jeunes graveurs comme l'avait fait le peintre en son temps ${ }^{4}$.

Après la mort de Raphaël en 1520, Raimondi demeura à Rome et travailla avec ses élèves, principalement Giovanni Francesco Penni et Giulio Romano. En 1524, la publication du recueil de gravures érotiques intitulé $I$ modi ${ }^{5}$ à partir desquelles l'Aretin composa les Sonetti fit scandale et le pape Clément VII le fit emprisonner; selon Vasari, le sculpteur Baccio Bandinelli intervint auprès d'Hippolyte de Médicis, futur cardinal, pour le sortir de cette situation. Trois années plus tard, le sac de Rome par les troupes du connétable Charles de Bourbon l'obligea à se réfugier à Mantoue puis à Bologne où il acheva misérablement sa vie ${ }^{6}$. Même si dans la Rome des années 15101520, le nom de Raimondi fut associé à celui de Raphaël, le graveur fut bien loin de n'avoir travaillé que d'après lui ${ }^{7}$. Outre ses copies de Dürer, il a gravé d'après Bandinelli et nombreuses furent les planches d'après Francesco Francia.

Les deux principaux élèves de Raimondi furent Augustin Vénitien et Marco Dente; né vers 1490, originaire de Venise ainsi que son surnom l'affirmait, Agostino Veneziano, de son vrai nom Agostino di Musi, a signé presque toutes ses estampes du monogramme A.V.; le goût de la gravure au burin lui est peut-être venu de Giulio Campagnola mais il a aussi beaucoup copié Dürer et son œuvre la plus anciennement datée $\left(1514^{8}\right)$ était une copie de la Cène. En 1515 il était à Florence où il grava notamment un Christ mort d'après Andrea del Sarto ${ }^{9}$ et une Cléopâtre d'après Bacio Bandinelli ${ }^{10}$. L'année suivante, il s'installa à Rome et entra dans l'atelier de Marcanto-

1. Les deux Massacres des Innocents, Sainte Cécile, le Martyre de sainte Cécile, le Jugement de Pâris, le Parnasse et la Peste de Phrigie.

2. TIB, vol. 27 , p. 49.

3. $T I B$, vol. 26, p. 11.

4. J. Shearman, Raphael in early modern sources, ouvr. cit., p. 1115.

5. Les dessins originaux étaient l'œuvre de Giulio Romano.

6. Raimondi mourut avant 1534, date de la Cortegiana, comédie de l'Aretin dans laquelle son nom est évoqué au passé.

7. Raimondi n'a pas gravé plus de cinquante planches d'après Raphaël et son élève Veneziano en a gravé davantage.

8. TIB, vol. 26, p. 40.

9. The Illustrated Bartsch, vol. 26, p. 56. S'il faut croire Vasari, éd. Chastel, t. 7, p. 75, le peintre, mécontent du travail d'Agostino, renonça à lui demander d'autres estampes. Mais Vasari a placé ce séjour après le sac de Rome.

10. TIB, vol. 26 , p. 190. 
nio qu'il quitta vraisemblablement pour travailler dans son propre atelier. La date de sa mort reste inconnue, la dernière date inscrite sur une de ses estampes étant $1537^{1}$. Au dire de Vasari, jusqu'à la mort de Raphaël, Veneziano travailla en collaboration avec Marco Dente ${ }^{2}$, appelé aussi Marc de Ravenne, né à Ravenne vers 1493 ; il était venu à Rome en 1515, vraisemblablement avant Veneziano et comme ce dernier il fut l'élève de Raimondi avant de travailler avec lui. Une seule estampe est datée, portant l'année 1519. Une tradition rapporte qu'il fut tué en 1527 dans l'atelier de son maitre, sous ses yeux, par les troupes du connétable de Bourbon. Ce buriniste signa ordinairement du monogramme $S R$, quelquefois du seul $R$. S'appuyant sur Benvenuto Cellini, Mariette assure qu'il commença sa carrière comme orfèvre ${ }^{3}$. Longtemps la signature $S R$ fut attribuée, en particulier par l'abbé de Marolles puis par le père Orlandi, auteur de l'Abecedario, à Sylvestre de Ravenne, graveur de pure invention, dont on chercherait vainement les estampes.

Tout en travaillant sur Raimondi dont l'œuvre a été examiné gravure après gravure, la conférence a porté la même attention aux estampes gravées d'après Titien. Comme Raphaël et presque concomitamment, Titien montra un grand intérêt envers les estampes et, sans jamais avoir gravé lui-même, non seulement il donna des dessins destinés à être gravées sur bois mais il dessina lui-même sur les planches de bois. C'est ainsi que nous comprenons l'estampe gravée par Johannes Breit, venu à Venise vers 1535 , qui italianisa son nom en Giovanni Britto ${ }^{4}$, dans laquelle Titien s'est représenté dessinant sur une planche de bois ${ }^{5}$. Le peintre ne s'intéressa à la taille-douce que plus tard, en 1565-1566, en appelant Cornelius Cort à venir travailler auprès de lui. L'importance qu'avait prise la gravure sur bois à Venise a conduit à attribuer à Titien de nombreuses compositions et nous avons porté sur elles un regard neuf, refusant par exemple La Vierge et l'Enfant entre saint Jean-Baptiste et saint Grégoire, estampe de Lucantonio degli Uberti dans laquelle nous avons reconnu trop d'emprunts ou de citations pris chez Raphaël ou chez Dürer pour lui en reconnaître la paternité. De même nous ne saurions affirmer qu'il avait procuré les dessins de La Vision des sept chandeliers d'or et des Quatre cavaliers, qui comme les treize autres illustrant une Apocalypse imprimée à Venise en 1515-1516 par Alessandro Paganini, copiaient celles de Dürer, même si La Vision offrait une composition différente mais difficilement attribuable à Titien. De même, on ne peut regarder comme ayant été dessiné par Titien le Déluge, gravé anonymement en deux planches, même si l'épreuve conservée au Cabinet des estampes ${ }^{6}$ porte l'inscription, manuscrite il est vrai, Titien inv. Si une première

1. TIB, vol. 27 , p. 93 .

2. Vasari, éd. Chastel, p. 72 : «En somme, Agostino et Marco, à eux deux, gravèrent à peu près tous les dessins et peintures de Raphaël, ainsi que plusieurs peintures de Jules Romain. ...Après sa mort [de Raphaël], Marco et Agostino se séparèrent et ce dernier se mit à travailler pour le sculpteur florentin Bacio Bandinelli...».

3. N. mss., t. 8, fol. 28 : «Benvenuto Cellini, dans son Traité de l'orfévrerie, pag. 2, fait mention de Marc de Ravenne et dit qu'il estoit orfèvre. . . Cellini a fait une observation qui est curieuse à l'égard de la graveure, il falloit presque la nécessité qu'elle fût exercée par des orfèvres ».

4. L'estampe est signée in Venitia par Giuovanni Britto Intagliatore.

5. L'estampe fut gravée en 1550 : une lettre de l'Aretin datée de juillet remerciait le graveur de lui avoir envoyé une épreuve.

6. BNF, Est., Bc 10, format 5 . 
notice des Notes manuscrites trouvait des raisons pour lui en attribuer l'invention ${ }^{1}$, une note en marge indique le doute d'un second rédacteur qui a attribué le dessin à un artiste florentin, peut-être Pontormo ${ }^{2}$. Quant à nous, nous sommes certain qu'il se trouve dans cette composition où chacun des personnages était montré dans une posture différente, une réminiscence de la Bataille de Cascina et nous refusons l'idée selon laquelle le dessinateur pourrait être Jan Van Scorel ${ }^{3}$ (1495-1562). On trouve dans Le Martyre des dix mille, autre estampe gravée sur bois, en huit planches, des citations de la Bataille de Cascina et de la voûte de la chapelle Sixtine. Auparavant le thème avait inspiré Dürer ${ }^{4}$ et Carpaccio, lequel en fit un tableau célèbre mais rien ne permet de considérer que Titien ait voulu interpréter à son tour le même thème.

Une des premières estampes dont il donna le dessin fut Le Cortège triomphal de la Foi, appelé aussi Le Triomphe du Christ. Citée par Vasari ${ }^{5}$ qui l'a datée de 1508 et vraisemblablement, s'est trompé, elle aurait été dessinée entre 1514 à 1516, Titien œuvrant pour le libraire vénitien Bernardo Benalio. Il apparaît en effet que ce dernier avait demandé le 6 mai 1516 un privilège pour éditer des estampes; parmi elles figurait une processionnal visione imaginaria del Salvatore nostro in foglio octo cum bellissimi ornamenti, mais il faut bien reconnaître que le projet me mentionnait que huit feuilles alors que complète, longue de 2,680 m et haute de 0,385 m., l'estampe en compte de dix. Dans sa forme, elle était inspirée par Le Triomphe de César de Mantegna. La source religieuse était un ouvrage de Jérôme Savonarole, Triumphus crucis... qui connut à Florence et à Venise de nombreuses éditions et qui, selon Vasari, avait inspiré à Botticelli une estampe ${ }^{6}$ dont on ne connaît plus d'épreuve. Repérer les éditions de celle de Titien n'est pas aisé ; celle en cinq planches éditée par Gregorio de Gregoriis copiait peut-être l'édition originale. La plus ancienne parvenue jusqu'à nous et imprimée avec les bois originaux est peut-être celle éditée par Lucantonio degli Uberti $^{7}$ conservée aux Offices. On ignore tout des modalités qui ont fait que les bois appartenaient en 1543 à Joos Lambrecht, éditeur d'estampes à Gand mais il est certain

1. N. mss., t. 9, fol. 89 : « ... sans aucun nom de peintre ni de graveur mais l'opinion commune est qu'elle est d'après le Titien et, en effect, elle est bien dessinée et dans son goust; aussy en a-t-il peut-être dessiné le traict sur le bois ».

2. N. mss., t. 9, fol. 89 : « J'avais toujours douté que ce déluge fût d'après le Titien et depuis que j'ai lu dans le père Orlandi qu'il y avait un déluge d'après le Pontorme, je suis très porté à croire que celui-ci est de ce maistre. C'est la manière de composer qui a été en usage chez les Florentins ; le Titien n'auroit point espacé ainsi ses groupes, il les auroit liés et leur auroit fait produire de l'effect que l'on voye [à] son passage de la Mer rouge. Qui a composé l'un peut-il estre regardé comme l'auteur de l'autre? ».

3. Catalogue de l'exposition The Genius of Venice, p. 329.

4. TIB, vol. 10, p. 212.

5. Vasari, éd. Chastel, t. 10 : «L'année suivante, en 1508, Titien publia une gravure sur bois, Le Triomphe de la Foi, avec une foule de figures, Adam et Ève, les Patriarches, les Prophètes, les sibylles, les Saints Innocents, les martyrs, les apôtres et Jésus-Christ assis sur un char de triomphe, porté par les quatre évangélistes et les quatre docteurs suivis par les quatre confesseurs : dans cette œuvre Titien montra un art énergique et un savoir-faire consommé. »

6. Vasari, éd. Chastel, t. 4, p. 261 : «Il fit de nombreuses estampes d'après ses desseins mais mauvaises car la gravure en était mal faites; la meilleure est le Triomphe de la Foi de frère Jérôme Savonarole de Ferrare. »

7. Elle a été copiée sur bois par Andrea Andreani. 
qu'il en publia des éditions qui différaient par les textes accompagnant les figures ${ }^{1}$. Les bois sont repérés ensuite à Anvers chez la veuve de Cornelis Lifrinck ${ }^{2}$.

Le Sacrifice d'Abraham était aussi une estampe faite de quatre bois assemblés. L'œuvre est assez bien documentée puisque le même libraire et imprimeur vénitien Bernardino Benalio obtint du sénat de Venise, le 9 février 1515, un privilège pour éditer, entre autres estampes, la hystoria del sacrifitio de Abraham. Le graveur qui a taillé les planches n'était autre qu'Ugo da Carpi dont le nom apparaissait sur les tirages de la première édition ${ }^{3}$ avec celui de Benalio ${ }^{4}$. En revanche le nom de Titien n'apparut que sur une édition tardive ${ }^{5}$ ce qui a conduit à douter qu'il fût bien l'auteur de la composition. Pourtant deux dessins attribués avec raison au peintre peuvent être rapportés à cette estampe. Le premier conservé au Louvre (inv. 4645) représente Abraham et ses serviteurs et, si un des personnages a été omis dans l'estampe, il est clair qu'il en constituait la source directe. Il a été dessiné tel qu'il était puisque, sur l'estampe, la composition était inversée. Le second dessin, conservé à New York, est un groupe d'arbres, cité deux fois dans la partie supérieure de l'estampe. L'estampe offrait en outre des similitudes avec les fresques de la vie de saint Antoine, peintes par Titien en 1511 dans la Scuola del Santo de Padoue. Toutes ces citations tendent à prouver que l'auteur du dessin n'était sans doute pas Titien lui-même mais un familier qui connaissait bien son œuvre et avait accès à son travail : peut-être Giulio Campagnola. C'étaitlà l'opinion de Mariette ${ }^{6}$ qui a décrit une édition avec une légende différente.

Imprimé à partir de douze bois assemblés en trois rangées de quatre qui lui conféraient des dimensions hors du commun (1,210 $\mathrm{m} \times 2,190 \mathrm{~m})$, Le Passage de la Mer rouge, est, avec le plan de Venise gravé en 1500, une des plus grandes xylographies jamais conçues et réalisées. Thème qui a fait naître peu de représentations, l'épisode biblique avait inspiré un bas-relief en bronze dû à Bartolomeo Bellano que Titien a pu voir dans la basilique San'Antonio de Padoue. Tous les exemplaires connus, complets des douze planches, portent le nom de Domenico della Greche qui se qualifiait de depentore venitiano et mentionnent le nom de Titien ${ }^{7}$ qui avait dessiné la composition directement sur les planches. Ils portent tous la date 1549 et on s'accorde à considérer

1. Le Cabinet des estampes de la BNF conserve (Bc 7 fol.) une édition de Joos Lambrecht avec les textes.

2. Le British Museum possède un tirage de cette édition.

3. On n'en connaît plus qu'une seule épreuve conservée au Staalische Museen de Berlin. La légende est rédigée ainsi In Venitia per Ugo da Carpi/ Stampata per Bernardino Benalio/ Cum privilegio concesso per lo illustrissimo senato.

4. Le nom de Benalio apparaît sur une autre édition, associé à celui de Bartolomio Bianzago. Se reporter plus bas à la note 6 .

5. Le Cabinet des estampes de la BNF conserve une épreuve du $2^{\mathrm{e}}$ (ou $3^{\mathrm{e}}$ ) état avec cette légende $S A C R I$ FICIO/DEL PATRIARCA/ ABRAHAM. Sur un état postérieur la mention DEL CELEBRE TIZIANO a été ajoutée.

6. N. mss., t. 9, fol. 116 : «L'estampe du sacrifice d'Abraham gravée en bois en quatre feuilles sur le dessein du Campagnole se trouve presque toujours de mauvaise édition. Les bonnes sont celles où on lit dans un cartouche au haut de la planche cette inscription en lettres gothiques La historia de Abraam, come por commendamento del signor Dio... Stampata in la christianissima citadi venitia per Bernardino Benalio et Bartolomio Bianzago compagni ».

7. « Designata per mano dil grande et immortal Titiano ». 
qu'ils appartiennent à une édition postérieure. La première édition ${ }^{1}$ avait été mise au jour en 1515-1516 et elle était le fait de Bernardo Benalio qui, en février 1515, avait sollicité des autorités vénitiennes la permission de publier un certain nombre d'estampes, parmi lesquelles se trouvait la Submersione di pharaone. Le sujet était biblique mais les estampes étaient peut-être chargées d'une signification politique. Pour actualiser la scène, des éléments contemporains ont été intégrés, ne serait-ce que les soldats et cavaliers revêtus d'armures du temps, et l'engloutissement de l'armée égyptienne était représenté devant une ville qui ne pouvait être que Venise, la cité s'étant trouvée dans une situation extrêmement critique après la défaite d'Agnadel en 1513.

Connu par une seule épreuve ${ }^{2}$, le Saint Jérôme en compagnie d'un ange, est un bois gravé anonymement mais vraisemblablement né d'un dessin de Titien comme l'ont affirmé les Notes manuscrites ${ }^{3}$. L'estampe porte la date 1515 et la scène représentée s'apparentait, semble-t-il, au songe qui visita le saint et par lequel le Christ lui faisait reprocher par un ange d'aimer davantage l'Antiquité que la foi chrétienne car il préférait lire Cicéron plutôt que les prophètes. On doit à Titien le dessin d'un autre Saint Jérôme ${ }^{4}$, postérieur au précédent et le montrant dans une autre iconographie; l'estampe passe pour être la première qu'exécuta Ugo da Carpi en chiaroscuro et fut imprimée au moyen de deux bois. Elle est signée $V G O$ et bas à droite et le nom du peintre, TICIANVS, y apparaît aussi.

L'estampe représentant saint Roch, l'un des patrons de Venise, averti par un ange qu'il était frappé par la peste ${ }^{5}$, doit être mise en corrélation avec la construction de la Scuola di san Rocco; elle a été gravée pour être vendue aux fidèles et les inciter à faire des dons ${ }^{6}$ : au bas, au-dessus de la légende, se voyait un tronc, appelé limosina per la fabrica (pour elimosina), en face duquel se trouvait un buste d'enfant, certainement un ex-voto qui témoignait de la guérison miraculeuse d'un enfant. Tout près, un second ex-voto montrait un malade guéri. Le dessin de la figure centrale du saint n'est qu'attribué à Titien et la représentation était complétée par huit bois qui racontaient en images des épisodes de sa vie. Il faut rapprocher cette estampe d'une autre qui représente Le Christ portant sa croix lequel, une corde passée autour de son cou ${ }^{7}$, est tenu par un bourreau. Peint en 1510 et toujours conservé à la Scuola di san Rocco, maintenant presque unanimement attribué à Titien, le tableau a fait l'objet d'une dévotion

1. Un fragment, conservé au musée Correr, est considéré comme appartenant à la première édition.

2. BNF, Est., Bc 7 fol.

3. N. mss., t. 9, fol. 97 : « On ne trouve à cette pièce aucun nom d'artistes mais je ne fais point de difficulté à la donner au Titien. Elle est dessinée d'un grand goût, les hachures qui forment les contours et les ombres y sont données avec un grand art et produisent un moelleux qu'il n'y a guerres de doute que le Titien qui l'ait commis. Cette pièce est gravée en bois. Elle est chez le Roy dans son œuvre du Titien ».

4. TIB, vol. 48 , p. 123 .

5. Sur l'iconographie de saint Roch, se reporter à l'étude d'Augustin Fliche, « Le problème de saint Roch », Mélanges Paul Peters (Analecta bollandiana), 1942.

6. C'était la conclusion qu'on peut lire dans les N. mss., t. 9, fol. 98 : « Aparemment que cette estampe fut faite lorsqu'on entreprit de bâtir la fameuse école de St-Roch et qu'on la distribua pour exciter les fidèles à cette bonne œuvre ».

7. N. mss., t. 9, fol. 98 : « Il est à remarquer que cette estampe fut faite lorsqu'on entreprit de bâtir la fameuse école de $\mathrm{S}^{\mathrm{t}}$-Roch et qu'on la distribua pour exciter les fidèles à cette bonne œuvre ». 
extraordinaire, des miracles ayant été attestés. On comprend donc que cette peinture ait fait naître une estampe qui, toute grossière qu'elle fût, nourrissait la piété des fidèles qui imploraient son intercession. La légende l'indique ${ }^{1}$, elle a été gravée en 1520. On n'en connaît plus qu'une seule épreuve conservée au cabinet des estampes ${ }^{2}$ de la BNF et c'est celle-là même qu'ont décrite les Notes manuscrites.

Sur une Adoration des bergers gravée en s'inspirant d'un tableau ${ }^{3}$ peint en 1532 1533 pour Francesco Maria della Rovere, duc d'Urbin, apparaît le monogramme de Giovanni Britto. L'estampe fut copiée en une taille-douce, de mêmes dimensions, éditée par Lucas Bertelli où on peut lire Tytianus pro Berteli.

Poursuivant l'étude des estampes sur bois gravées d'après Titien, la conférence a prêté toute son attention à un des monuments intellectuels du XVI ${ }^{\mathrm{e}}$ siècle européen, le De humanis corporis fabriqua d'André Vésale auquel ont vraisemblablement participé le peintre ou quelque membre de son atelier; il a été publié chez l'imprimeur Johannes Operinus à Bâle en 1543. Il a été rappelé aux auditeurs que dans la première moitié du siècle, les estampes avaient été indispensables à l'essor de deux sciences, la botanique et la zoologie, parce qu'elles procuraient des images de plus en plus véridiques qui, circulant facilement, ont permis la diffusion des nouvelles connaissances. Plus tardivement, il en fut de même pour l'anatomie et Vesale (1514-1564) eut recours aux images pour illustrer ses observations et faire approuver ses idées. Avant la Fabrica, il avait publié en 1538 à Venise, les Tabula anatomica sex, en six planches, premiers fruits de ses travaux anatomiques. Toujours chez Oporinus et la même année que la Fabrica, Vésale publia l'Epitome, où se trouvaient onze planches gravées sur bois mais différentes de celles de la Fabrica.

De l'aveu même de Vésale, les trois premières planches des Tabulae étaient son œuvre, les autres étant celle de Jan Stephan Calchar ( $c a$ 1499-1546/1550), élève de Titien à partir de 1536-1537. Les planches de la Fabrica furent gravées à Venise, ceci se lit dans la préface, peut-être par Francesco Marcolini de Forle, grande figure de l'édition vénitienne de 1530 à 1550 qui, au dire de l'Arétin, avait la réputation d'être un graveur en bois très talentueux. Le fait que Calchar était l'élève de Titien a conduit à considérer que le peintre n'avait pas été étranger à l'élaboration des dessins de la Fabrica, supérieurs à ceux des Tabulae. Cette opinion s'est trouvée confortée par le fait qu'en 1670 Dominicus Bonavera publia un recueil intitulé Notonomia di Titiano composé de planches copiées sur celles de la Fabrica où fut inscrit le monogramme TID, compris comme signifiant Titianus Invenit Delineavit. Cependant nous pensons qu'il faut nuancer cette proposition et considérer que la participation de Titien fut seulement d'avoir repris les croquis de Vésale pour les traduire en dessins prêts à être gravés : les trois célèbres squelettes, rendus vivants par la qualité du dessin et par leur posture, étaient loin d'être de simples croquis anatomiques. D' autre part, les paysages placés derrière les écorchés étaient manifestement inspirés de ceux de Titien ou de son

1. Figura del deuotissimo e miraculoso Christo en ella chiesa del deuotoSan Rocho di Venitia. B CCCCC $X X$.

2. BNF Est., Bc 6 fol.

3. Ainsi le paysage à l'arrière plan copiait un dessin conservé à Francfort, Paysage avec les deux astrologues. Une copie du tableau est conservée à Oxford. 
atelier. Quant au dessin du titre illustré ${ }^{1}$ représentant une démonstration anatomique pratiquée dans l'amphithéâtre de Padoue et attribué à Titien, il nous semble proche de la manière de Giulio Campagnola. Enfin s'il est vrai que le portrait de Vésale en tête de la Fabrica était l'œuvre de Calchar, on ne peut lui attribuer les deux planches de l'Epitome montrant un homme et une femme debout, nus et vus de face. La qualité propre à ces deux dessins nous conduit à y voir l'invention de Titien.

Le peintre ne s'est-il intéressé aux tailles-douces que dans les dix dernières années de sa vie, soit à partir de 1565-1566, lorsque le graveur flamand Cornelius Cort ${ }^{2}$ (1533-1578) est venu travailler auprès de lui ? Presque trente ans auparavant, Gian Giacomo Caraglio avait gravé, au plus tard en $1538-1539^{3}$, une Annonciation ${ }^{4}$ peinte pour le couvent Santa Maria degli Angeli à Murano dont les religieuses avaient refusé de payer les 500 écus demandés par le peintre ${ }^{5}$. L'estampe déplut-elle à Titien ? Avaitelle été gravée sans son accord? Toujours est-il que ce fut seulement en 1563 que Giulio Bonasone ${ }^{6}$ grava d'après lui, à l'eau-forte et au burin, une série d'estampes : une Vierge à l'Enfant ${ }^{7}$ dont le modèle est perdu mais dont l'attribution à Titien est certaine $^{8}$, un Christ au jardin des oliviers ${ }^{9}$ dont la lettre indiquait Titiano Inventor Ivlio Bonasone $F$., et qui fut copié par Nicolas Beatrizet ${ }^{10}$, l'estampe étant éditée par Lucas Bertelli, une Mise au tombeau ${ }^{11}$ où étaient inscrits les noms du peintre, du graveur et la date ${ }^{12}$, et un Christ en croix, sans doute peint par Orazio, fils de Titien, mais dont celui-ci avait donné le dessin. Bonasone a fait toute sa carrière à Rome et si Titien y a séjourné en 1544, il nous est difficile de discerner les voies par lesquelles le graveur a rencontré les œuvres du peintre, les plus vraisemblables étant des copies des dessins.

Avant la venue de Cort à Venise, un autre tableau Le Christ interrogé sur le tribut dû à l'empereur ${ }^{13}$ fut traduit en une estampe gravée par Domenico Campagnola ${ }^{14}$, au plus tard en 1564 ; vraisemblablement il avait œuvré d'après un dessin, le tableau ayant été envoyé à Philippe II en 1568. L'estampe ${ }^{15}$ était éditée chez Lucas Bertelli. Un gra-

1. Conservé à la bibliothèque Huntington à San Marino en Californie.

2. Ce graveur a fait l'objet de deux principaux travaux : celui de Bierens de Haan (J. C. J.), L'auvre gravé de Cornelis Cort, graveur hollandais, 1533-1578, La Haye, 1948, et les 3 volumes qui lui sont consacrés dans la suite du New Hollstein, Dutch and flemish etchings, engravings and woodcuts, 2000.

3. Le tableau était achevé en novembre 1537 (lettre de l'Aretin à Titien du 7 novembre). D'autre part, Caraglio partit pour Cracovie en 1539 où il mourut le 26 août 1565.

4. TIB, vol. 28, p. 79 .

5. Titien en fit don à l'impératrice Isabelle ou à l'empereur lui-même.

6. Né vers 1498-1510 à Bologne, mort en 1574. Cf. Stefania Massari, Giulio Bonasone, Rome, 1983.

7. TIB, vol. 28 , p. 271.

8. L'œuvre a également été gravée par Martin Rota et, au XVII ${ }^{\mathrm{e}}$ siècle, par Valentin Lefèbvre qui a inscrit cette lettre Tizianus Vecellius, cad. Invent. \& Pinxit.

9. TIB, vol. 28, p. 244. La tableau, peint en 1563, est conservé à l'Escurial de Madrid.

10. $T I B$, vol. 29 , p. 262.

11. TIB, vol. 28, p. 248. Il s'agit de la seconde Mise au tombeau, peinte avant 1559; la première, conservée au Louvre a été exécutée vers 1526/1532 et n'a été gravée qu'au XVII ${ }^{\mathrm{e}}$ siècle par Gilles Rousselet.

12. TITIANO INVENTOR I BONASONE 1563.

13. Le sujet a été peint une première fois par Titien en 1516 et une seconde fois en 1568 .

14. Une épreuve s'en trouve dans le recueil du Cabinet des estampes.

15. Cette estampe a été copiée par Martin Rota (TIB, vol. 33, p. 13), elle-même copiée par Cornelius $I^{\text {Ier }}$ Galle (Anvers 1575-ibid.. 1650). 
veur resté anonyme exécuta pour le même éditeur une Cène; si le nom de Titien est inscrit sur l'estampe, la composition ne ressemble à aucune des deux toiles du peintre connues mais il en avait peint une troisième détruite en 1571. L'estampe en était-elle la traduction? Construite non pas dans la longueur mais dans la hauteur, elle offrait quelques similitudes avec la peinture conservée à l'Escurial (Judas et l'apôtre à sa gauche, celui à la gauche du Christ étaient les mêmes), mais les lourds rideaux, la présence du Saint-Esprit au milieu d'une gloire, les servantes qui apportaient les plats, faisaient oublier l'essence même de la représentation, la trahison de Judas. Faut-il considérer comme gravée d'après Titien une Flagellation ${ }^{1}$, estampe de Giovanni Battista ${ }^{2}$ Franco, gravée au plus tard en $1561^{3}$ et copiée par Martin Rota ${ }^{4}$ en 1568 ? Les Notes manuscrites n'en doutaient pas ${ }^{5}$, même si ni l'estampe originale ni la copie n'en faisaient état. Autre estampe, gravée avant 1565 (si elle était l'œuvre de Nicolas Beatrizet mais elle est attribuée aussi à Cornelius Galle), celle de la Transfiguration ${ }^{6}$.

Nous doutons que ces premières tailles-douces aient été gravées avec l'accord de Titien et nous pensons qu'à partir de 1565 il entendit avoir l'œil sur les estampes faites d'après ses œuvres. À la fin de cette année, Cornelius Cort arrivait à Venise et habita chez le peintre à qui Lampsonius l'avait recommandé. Après un séjour d'un an, Cort partit pour Rome où il travailla pour différents éditeurs d'estampes puis, après un passage à Florence, il revint travailler près de Titien. De nouveau il quitta Venise pour Rome en 1572 où il mourut. Avant son premier séjour vénitien, Cort avait eu l'occasion de rencontrer l'art de Titien et il avait gravé un Mariage mystique de sainte Catherine, non pas d'après le tableau peint pour le tombeau des Trevisani dans l'église Santi Giovanni e Paolo, ni même d'après un hypothétique dessin mais d'après l'estampe en bois attribuée à Nicolo Boldrini (1510-après 1566), différente du tableau par la présence de saint Joseph et de deux anges, dont la lettre du deuxième état indiquait Titianus vecellius inventor lineavit. Plus petite et anonyme, inversée et mise en hauteur, 1'estampe de Cort reprenait une partie de la signature?

Aucun tableau ou dessin représentant Joseph et la femme de Putiphar n'est répertorié dans l'œuvre de Titien mais, en dépit de la différence stylistique des deux personnages, la lettre de l'estampe gravée par Cort indiquait clairement que son modèle était Titien $^{8}$. Le modèle de la Putiphar doit être cherché dans une Danaë ou une Léda, tandis que le corps de Joseph montrait le modelé d'un corps en mouvement. La réunion des deux personnages pour illustrer l'épisode biblique n'était peut-être pas l'œuvre de Cort et il est possible qu'il ait été abusé.

1. Aucune peinture ni dessin de Titien ne correspondent à cette composition. Mais Vasari, éd. Chastel, t. 10, p. 34, a mentionné un Christ à la colonne peint pour la reine du Portugal et, dans une note, l'éditeur du texte assure que cette toile n'est plus connue que par l'estampe de Rota.

2. TIB, vol. 32, p. 166.

3. C'est l'année de la mort de Giovanni Battista Franco, né vers 1510 à Venise.

4. TIB, vol. 33, p. 15. L'estampe était éditée par Lucas Bertelli.

5. N. mss., t. 9, fol. 90 : « Jésus-Christ attaché à la colonne et fouetté par les bourreaux. Gravé à l'eau-forte et terminé ensuite au burin par Baptiste Franco peintre vénitien... Le nom de Titien n'y est pas, elle est cependant d'après luy. Il y en a une petite estampe de M. Rota. »

6. BNF Est., Bc 6 fol. L'estampe est inversée.

7. Tycianus inventor.

8. TYCIAN INVE. 
Une des premières estampes que le graveur fit à Venise sous la direction de Titien fut un Saint Jérôme ${ }^{1}$ dont la lettre indiquait qu'elle avait été gravée d'après Titien et à Venise ${ }^{2}$. Vint ensuite une Sainte Marguerite d'après celle peinte ${ }^{3}$ vers 1560 pour Marie de Hongrie, sœur de Charles Quint. L'estampe a été éditée par Lucas Bertelli, éditeur et marchand d'estampes vénitien et le nom de Titien n'apparaissait que sur le second état ${ }^{4}$. Elle a été gravée avant que Titien ait obtenu un privilège pour protéger les estampes nées de ses inventions. Il en fut de même pour une Annonciation, peinte vers 1560-1565 et toujours conservée en l'église San Salvadore; inversée, l'estampe de Cort ignorait le nom de Titien mais elle a été copiée en taille-douce à l'initiative de Lucas Bertelli qui y a ajouté le nom du peintre, puis anonymement sur bois.

Une première fois, vers 1528-1530, et pour la confrérie installée dans l'église Santi Giovanni e Paolo, Titien a peint Le Martyre de saint Pierre Martyr, sujet peint de nouveau en 1567-1568. Si les deux tableaux sont maintenant détruits ou perdus, ils ont été l'un et l'autre gravés mais nous n'avons retrouvé aucune estampe qui fût de Cornelius Cort. Le premier a fait l'objet de deux planches, l'une due à Giovanni Battista Fontana (1525-1587), la seconde à Martino Rota (ca 1520-1583), les deux dans le même sens, indiquant le nom du peintre et peut-être exécutés du vivant de Titien. Le second tableau ${ }^{5}$, offert par Titien au pape Pie $\mathrm{V}$, a fait naître une gravure anonyme attribuée à Cort par Bierans de Haan, à Casparo Oselli ${ }^{6}$ par les Notes manuscrites et classée dans l'œuvre de Martin Rota dans The Illustrated Bartsch'.

La première estampe pour laquelle Titien demanda et obtint lui-même un privilège le 4 février 1566 fut celle gravée d'après un dessin ou esquisse du célèbre tableau $L a$ Gloire de la Sainte Trinité, commandé par Charles Quint lui-même en $1550^{8}$ à propos duquel Vasari a écrit « on s'attend à voir bientôt ce chef-d'œuvre gravé » ${ }^{9}$. L'estampe est encore documentée par une lettre de Lampsonius à Titien, du 13 mars 1567, pour le remercier de lui avoir envoyé six estampes dont la Gloire de la Trinité et par une autre du 15 juin 1567 de Titien à l'infante Marguerite où il affirmait avoir fourni un dessin à Cort. Dans le même sens que le tableau, l'estampe est connue en quatre états. Le premier ne portait que le nom de Titien, la mention du privilège reçu et la date 1566 . Le nom du graveur n'apparaissait que sur le second état, avec son prénom italianisé en Cornelio $^{10}$. C'est le même schéma qui a prévalu pour un Saint Jérôme gravé en $1565^{11}$ et pour une Sainte Madeleine en pénitence copiée par Martin Rota qui, outre son nom, l'a signée

1. New Hollstein, vol. Cornelius Cort, t. 2, p. 158-159.

2. In Venetia Cor. Cort f., et Titiano inv.

3. Elle est conservée au musée du Prado à Madrid.

4. Titiani Vecelli Aquitis Cae. Regince Marice Imp. Caroli V. sororis opus.

5. Il n'est connu que par une lettre de Titien et différait du premier en ce que le saint écrivait sur le sol Credo.

6. Surnommé Patavinus ab avibus, né vers 1536 , mort après 1585 , il fut l'élève de Giorgio Ghisi.

7. TIB, vol. 33, p. 27.

8. La toile est conservée au musée du Prado à Madrid.

9. Vasari, éd. Chastel, t. 10, p. 32.

10. L'estampe de Cort a fait naître de nombreuses copies en taille-douce et une sur bois. Cf. New Hollstein, vol. Cornelius Cort, t. 2, p. 39-43.

11. Pour être exact, le $1^{\text {er }}$ état ne porte que la date, le $2^{\mathrm{e}}$ le nom du peintre et le privilège, le $3^{\mathrm{e}}$ le nom du graveur. 
d'une tablette semblable à celle de Raimondi ${ }^{1}$. Dernier thème inspiré par la religion, $L e$ Martyre de saint Laurent, a fait naître deux tableaux de Titien, le second tableau ayant été commandé par Philippe II pour le maître autel de l'Escurial ${ }^{2}$; pour des raisons que nous ignorons (destruction de la première planche ? usure due à son succès ?), cette composition fut gravée deux fois par $\operatorname{Cort}^{3}$ en $1571^{4}$, avec des différences infimes, d'après un dessin fourni par le peintre. Bien qu'elle fût la copie inversée des deux premières, une troisième planche, passe pour être également l'œuvre de Cort, mais nous n'en sommes guères convaincu. D'autre part Les Saintes Femmes devant le tombeau vide du Christ tableau peint par Titien en 1546, maintenant perdu, a fait naître une estampe en bois ${ }^{5}$ et

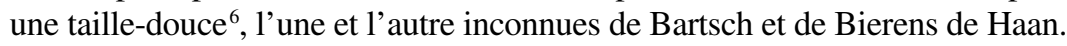

Toujours d'après Titien, cinq estampes à sujet profane ont été gravées par Cornelius Cort : Roger délivrant Angélique, Le supplice de Tityos, Diane et Callisto, Tarquin et Lucrèce et Les Cyclopes forgeant des armes. Gravé en 1565, Roger délivrant Angélique était inspiré du Roland furieux de l'Arioste, ami du peintre. C'est une des premières estampes faites par Cort à Venise et les Notes manuscrites croyaient pouvoir observer que Cort avait cherché dans le paysage, « à se défaire d'une mauvaise manière qu'il avoit prise chez luy pour prendre la belle manière de feuille du Titien $^{7}$ ». Trois dessins correspondent à cette estampe ; celui du Louvre qui a appartenu à Jabach ${ }^{8}$, est inversé tandis que celui conservé au musée Bonnat de Bayonne qui fit partie des collections de Mariette ${ }^{9}$ est dans le même sens ; faussement attribué à Domenico Campagnola, un troisième, également inversé, fait partie de la collection du duc de Devonshire. Il semble bien que le dessin original était celui ayant appartenu à Jabach ${ }^{10}$, celui du duc de Devonshire en étant une copie tandis que celui de Mariette copiait l'estampe. Le supplice de Tityos ${ }^{11}$ faisait partie d' un ensemble de quatre tableaux représentant des damnés, commandés à Titien par Marie de Hongrie en $1548^{12}$ et, l'année suivante, ils étaient dans sa résidence de Binche. Cort grava d'après un dessin, peut-être celui conservé au Louvre ${ }^{13}$ qui avait appartenu à

1. Les mêmes signatures se retrouvent sur une autre Madeleine gravée par Rota, manifestement inspirée de celle de Titien.

2. Bâti en souvenir de la prise de Saint-Quentin le 10 août 1557 , jour de la fête du saint.

3. New Hollstein, vol. Cornelius Cort, t. 2, p. 176-184.

4. La $1^{\text {re }}$ planche était achevée avant le $1^{\text {er }}$ août 1571 , date d'une lettre par laquelle Titien affirmait qu'il en avait envoyé deux épreuves à Philippe II.

5. Conservée aux Uffizi à Florence.

6. Conservée à Rome dans la collection Pio.

7. N. mss., t. 9, fol. 102 .

8. Cf. Bernadette Py, Everhard Jabach collectionneur. 1618-1665 : les dessins de l'inventaire de 1695, Paris, 2002.

9. N. mss., t. 9, fol. 102 : « J'ay le dessin même du Titien sur lequel cette estampe a été gravée; il est de la plus grande beauté. » Le dessin a été catalogué dans la vente Mariette sous le $\mathrm{n}^{\circ} 777$.

10. On sait que Jabach fit graver à l'eau-forte quelques-uns de ses dessins de Titien et que les estampes furent réunies en un recueil.

11. On y a reconnu aussi un Prométhé et c'est ce héros que présentait l'estampe d'Étienne Delaune copiée sur celle de Cort.

12. Les trois autres tableaux représentaient les supplices de Sisyphe, de Tantale et d'Ixion. La source littéraire était les Métamorphoses, liv. IV, v. 455-461. Seuls subsistent ceux de Tityos et de Sisyphe.

13. Inv. 5518. 
Mariette ${ }^{1}$. Dans les trois états de cette estampe, copiée par Lucas Bertelli et par Martin Rota $^{2}$, se retrouvent les mêmes caractéristiques observées précédemment : le nom du peintre, son privilège et la date 1566 apparaissaient sur le premier état tandis que le nom du graveur, italianisé, n'était que sur le second; quant au troisième, postérieur, il portait le nom de Stefano Scolari, graveur sur bois et marchand d'estampes vénitien. Le tableau du Tantale n'est plus connu que par l'estampe de Giulio Sanuto ${ }^{3}$, nécessairement gravée ${ }^{4}$ comme la précédente d'après un dessin de Titien, peut-être après sa mort.

Les personnages mythologiques de Diane et Callisto ont fourni à Titien le sujet de deux tableaux, l'un peint vers 1555-1559, le second en 1566. La source littéraire était un passage des Métamorphoses, à partir du vers 455 du livre II. Datée de 1566, l'estampe de Cort ne correspondait à aucun des deux tableaux; une nouvelle fois, Titien a fourni un dessin destiné à être gravé et les épreuves se répartissaient en trois états, le nom du graveur n'étant inscrit que sur le deuxième et celui de Scolari sur le troisième. Tirée de l'Histoire romaine de Tite-Live, des Fastes d'Ovide ou encore du De claris mulieribus de Boccace, l'histoire de Lucrèce fut souvent représentée; Titien le peignit entre 1568 et 1571 et, une nouvelle fois, Cort grava un dessin légèrement différent du tableau. Datée de 1571, l'estampe a fait naître de nombreuses copies. La dernière estampe gravée par Cornelius Cort représentaient Les Cyclopes forgeant des armes et elle demeure le seul document qui fasse connaître un des trois grands tableaux commandés à Titien en 1564 pour décorer le plafond du palais de Brescia, achevés en 1568 et détruits en 1575 .

La fin de l'année universitaire nous ayant contraint à mettre un terme aux travaux sur les estampes d'après Titien, la dernière conférence a été consacrée à une gravure ${ }^{5}$ en bois attribuée à Nicolo Boldrini ${ }^{6}$ et caricaturant le groupe sculpté du Laocoon, découvert à Rome en 1506 et considéré comme la quintessence de la sculpture antique. Sacrilège contre l'Antiquité vénérée, Lacoon et ses fils y étaient travestis en singes et Titien passa pour l'avoir inspirée. Incomprise de Mariette ${ }^{7}$, cette caricature ne pouvait, en son temps, être anodine et, pour en saisir le sens, on l'a rapprochée de l'emblème ${ }^{8}$ du peintre composé d'un ours, du Temps et de la Renommée, accompagnés de cette devise Natura potentior ars. La caricature doit-elle être regardée comme une sorte de manifeste esthétique du peintre, signifiant que ceux qui voulaient imiter la nature ne faisaient souvent que la singer et que l'artiste devait dépasser la simple imitation?

1. N. mss. t. 9, fol. 101 : « Prométhée attaché sur le mont Caucase où il est déchiré par un vautour. Cette excellente pièce a été gravée par Corneille Cort en 1566 sous la direction de Titien. J'en ai un dessin original de Titien et qui est de toute beauté, aussy savamment dessiné que l'auroit pu Michel-Ange. »

2. TIB, vol. 33, p. 115 .

3. Graveur vénitien, né vers 1520 , actif de 1540 à 1580 .

4. BNF Est., Bc 8 fol.

5. Gravée sur bois et attribuée à Nicolo Boldrini.

6. Elle a été copiée au burin avec le nom de Titien.

7. N. mss., t. 9, fol. 104 : «Un singe et ses deux petits environnés de serpents, dans l'invention du célèbre grouppe de Laocoon et de ses deux enfans qui est à Rome. Gravé au burin par un anonyme. On ne comprend pas quelle a été l'intention de Titien en produisant ce dessein burlesque [qui] tourne en ridicule et déprise un morceau de sculpture qu'il estimoit... Une autre planche de ce mesme dessein, gravée en bois. À celle-ci qui est certainement l'original, il n'y a aucun nom d'artiste et l'on ne comprend pas bien quelle a pu estre l'idée capricieuse du Titien en faisant ce dessein. »

8. Connu grâce à Ludovico Dolce. 